\title{
A Sensor Made from A Paste Electrode of Graphite Particles Grafted with Molecularly Imprinted Polymer
}

\author{
Yasuo YOSHIMI, Rina YAMAGUCHI, Maki SEKI \\ 3-7-5 Toyosu, Koto-ku, Tokyo 135-8548, Japan
}

\begin{abstract}
:
A real-time heparin monitor could be used to optimize the dosage of heparin during extracorporeal circulation procedures. This report describes the development of a graphite-paste (GP) electrode with molecularly imprinted polymer (MIP) grafted onto it. Heparin-imprinted poly

(methacryloxyethyltriammonium chloride -co- acrylamide -co- methylenebisacrylamide) was grafted directly onto graphite particles. The grafted particles were thoroughly mixed with oil to fabricate the MIP-GP electrode. Traditional cyclic voltammetry was performed with the electrode in physiological saline or bovine whole blood containing $5 \mathrm{mM}$ ferrocyanide and 0-8 units $/ \mathrm{mL}$ heparin. The current intensity increased with heparin concentration, due to expansion of the effective surface area resulting from heparin-promoted mobility of the oil in the MIP-GP electrode. The MIP-GP electrode, which operated through a new heparin-sensing mechanism, is an excellent candidate for application as a disposable sensor to monitor heparin levels in blood.
\end{abstract}

Key words: Molecularly Imprinted Polymer, Heparin, Graphite Paste Electrode, Redox, Surface Tension

\begin{abstract}
Introduction
In extracorporeal therapy, blood contacts artificial materials in the blood vessels, creating a risk of clotting. A sensor that could detect heparin, including $\mathrm{LMWH}$, in real time is needed to monitor heparin dosage. In the present study, a new method to improve the quality of MIPgrafted electrodes was developed by using a carbon pasting method that allowed mass production of the electrodes with high homogeneity. First, the graphite carbon particles were coated with a radical polymerization photoinitiator, and this was followed by radical polymerization to graft the MIP onto the particles. Finally, the grafted graphite was mixed thoroughly with oil to fabricate homogenous carbon paste electrodes. The heparin-sensitivity and the reproducibility of the output current at the MIP-grafted graphite paste (MIP-GP) electrode were evaluated in whole blood and in saline. The sensing mechanism of the MIP-GP was examined using the sensitivity of the contact angle of saline on the MIP-GP electrode to the presence of the template.
\end{abstract}

\section{Materials and Methods}

The diethyldithiocarbamate methylene group, which is a radical photopolymerization initiator, was introduced onto the surface of graphite particles. The MIP of heparin was grafted on the initiator-coated particles by irradiation to the particles fluidzed in a mixed solution of methacryloxyethyltriammonium chloride, acrylamide, methylenebisacrylamide and heparin. A non-imprinted polymer (NIP) was also prepared using the same procedure described above, but without the heparin template. The grafted particles were thoroughly mixed with oil to prepare a paste. The paste was packed a tip of a glass capillary to fabricate the MIP-GP electrode. Traditional cyclic voltammetry was performed with the electrode in physiological saline or bovine whole blood containing $5 \mathrm{mM}$ ferrocyanide and $0-8$ units $/ \mathrm{mL}$ heparin or chondroitin sulfate C (CSC), which is analogue of heparin. The sensitivity of the peak current to the heparin or the CSC was evaluated. The effect of heparin or CSC on the contact angle of a droplet of the physiological saline at the surface of MIP-GP was observed in order to speculate the sensing mechanism of the MIP-GP electrode.

\section{Results and Discussion}

The anodic current of the ferrocyanide at the MIP-GP electrode increased with the increase 
of heparin concentration but was insensitive to the CSC concentration as shown Fig. 1. The current at the NIP-grafted GP electrode was insensitive to heparin. The non-imprinted. The contact angle of physiological saline droplet on the MIP-GP was decreased by the heparin but was insensitive to CSC as shown Fig. 2. The contact angle at the NIP-GP surface was insensitive to heparin. The result indicates that the surface of MIP-GP is hydrophilized by the specific interaction with the template. The hydrophlization would be occurred by migration of the oil into the bulk of the MIP-GP [1]. The migration is thought to enhance the effective surface area of the MIP-GP electrode and increase the redox current. The current sensitivity at the MIP-GP would be applicable for disposable sensor due to the high cost performance and high reproducibility of carbon paste electrodes.

\section{References}

[1] Y. Yoshimi, Y. Yagisawa, R. Yamaguchi, M. Seki, Sensors and Actuators B, 259, 455-462 (2018): doi: 10.1016/j.snb.2017.12.084

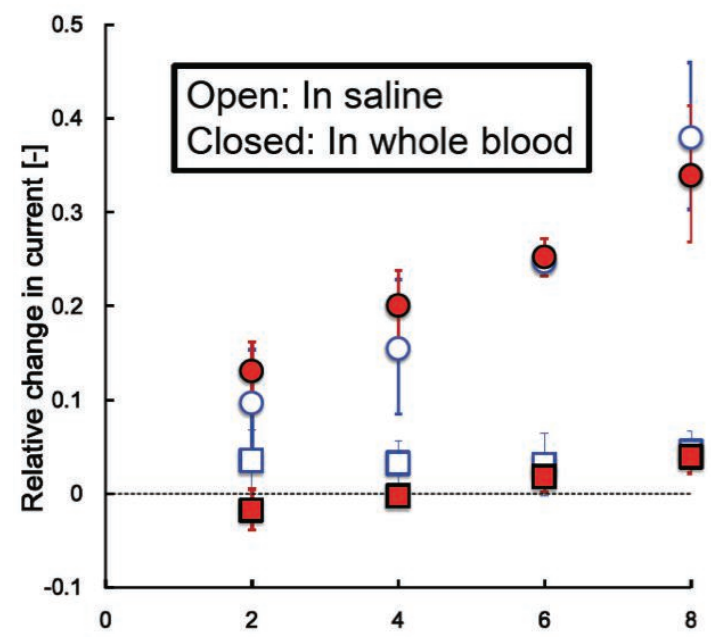

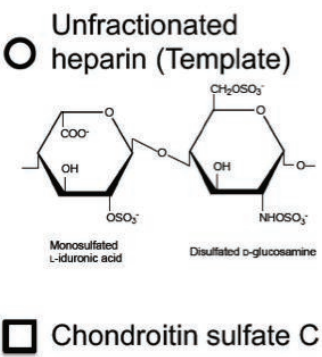

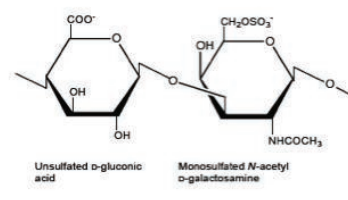

Fig 1. Relative change in anodic peak current of $5 \mathrm{mM}$ hexacyanoferrate (II) at the MIPGP electrode upon addition of unfractionated heparin (circles) and CSC (squares) in whole blood (closed) or physiological saline (open).

(A) Advancing

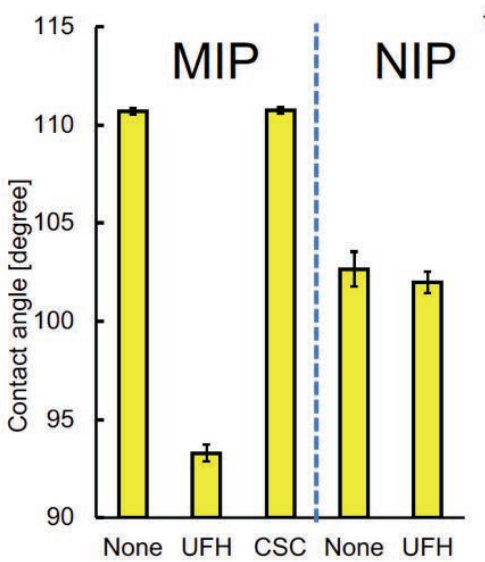

(B) Receding

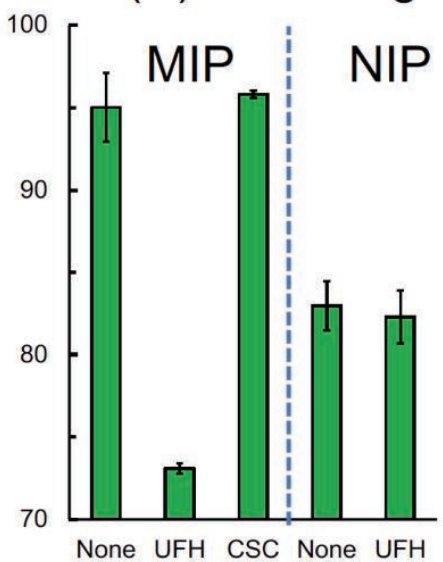

Fig 2. Effect of the presence of unfractionated heparin (UFH) or CSC (10 units $/ \mathrm{mL}$ ) on (A) advancing and (B) receding contact angle of physiological saline on MIP-GP or NIPGP. 